\title{
Reality bites of spider bites: a case report and review of the local epidemiology
}

\author{
A Visser, HF Visser
}

Adele Visser, MBChB, DTM\&H, PGDipTM, MMedClinPath, FCPathSA(ClinPath) Head of Division, Clinical Pathology, Department of Medical Microbiology, University of Pretoria, Pretoria Tshwane Academic Division, National Health Laboratory Services, Pretoria Hilgaard Visser, MBChB, MMedOrtho Orthopaedic Surgeon, Eugene Marais Life Hospital, Pretoria E-mail: adele.vis@gmail.com

\section{Introduction}

Spider bites are a frequent complaint, often prompting patients to seek medical attention. ${ }^{1}$ However, the diagnosis of a spider bite is frequently inferred by the patient, and a thorough evaluation subsequently reveals an alternate diagnosis. Gertsch et al demonstrated that of 600 consecutive "spider bite" cases, $80 \%$ were incorrectly diagnosed. ${ }^{2}$ Alternative diagnoses included bites by arthropods other than spiders, ${ }^{2}$ bacterial, viral or fungal infections ${ }^{3}$ and malignant tumours. ${ }^{3}$ In this study, we describe the case of a patient who was initially misdiagnosed as having been bitten by a spider. Later, the patient was demonstrated to have a spontaneous, soft tissue Staphylococcus aureus infection. We further describe the local epidemiology of cases presenting with spider bites and the subsequent microbiological findings.

\section{Case report}

Mr T, a 48-year-old man, presented with a five-day history of a septic spider bite on his right thigh. A diagnosis of a spider bite was made by the patient. However, he did not see the spider biting him. The wound showed an area of central necrosis with pus draining from the site, and a circumferential area of erythema (Figure 1).

Formal debridement was performed by the orthopaedic surgeon, during which time three tissue samples and three swabs were submitted for microbiological culture and sensitivity. Following debridement, the patient was treated with oral co-amoxyclav for five days. The wound healed completely in this time.

Culture results yielded methicillin-sensitive $S$. aureus in all six submitted samples. Based on this finding, and because the patient never saw a spider bite him, the diagnosis was revised

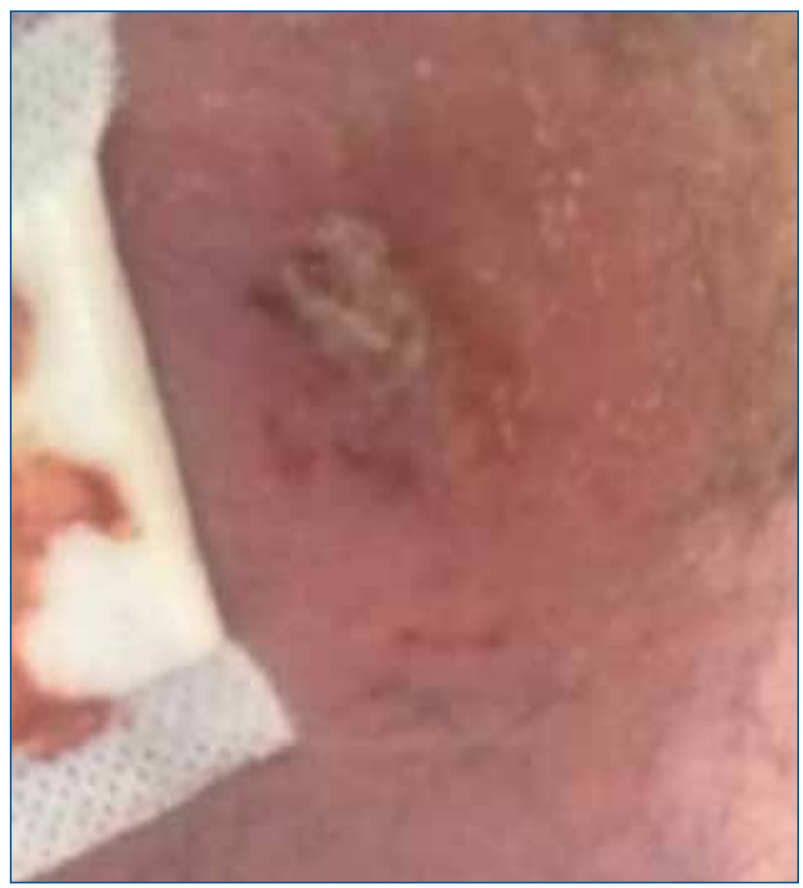

The lesion shows an area of central necrosis with surrounding erythema

Figure 1: Soft tissue infection, reported as a spider bite

to be a soft tissue infection due to $S$. aureus, rather than a spider bite.

\section{Method}

\section{Patient population}

Patients admitted to the Steve Biko Academic Hospital, Pretoria, who were reported to have been bitten by a spider between January 2006 and April 2012, a period of 78 months, and who were taken to theatre for debridement, were included 
in this study. Collected clinical data included the site of the lesion and demographic information.

\section{Microbiological sampling, isolation and sensitivity}

Samples had been submitted for microbiological analysis in all of the cases, and included culture and sensitivity, on either tissue samples or pus swabs. Current practice at Steve Biko Academic Hospital is to obtain multiple swab and/or tissue samples from a single patient during the initial debridement, irrespective of the number of lesions, in order to obtain a representative view of the microbiological pathogens involved.

The samples were inoculated onto blood, chocolate and MacConkey agars to enable culture of Gram-positive fastidious, and Gram-negative, organisms, respectively. Tissue samples were also subjected to anaerobic culture on $10 \%$ sheep blood agars. Organisms were identified utilising standardised microbiological methods. Gram-negative organisms were established using the Vitek $^{\circledR} 2$ system (BioMerieux, Johannesburg, South Africa). Gram-positive organisms were determined by employing DNase ${ }^{\circledR}$ plates for Staphylococcus spp. and the Remel Streptex ${ }^{\circledast}$ Rapid Latex Agglutination Test (Thermo Scientific, Johannesburg, South Africa) for Streptococcus spp. Sensitivity data were collected for all isolates, as determined per Clinical and Laboratory Standards Institute guideline methodology. ${ }^{4}$
This study was approved by the Research Ethics Committee of the University of Pretoria, protocol number 58/2009.

\section{Results}

\section{Patient population}

In total, 13 patients were included in this study, including $\mathrm{Mr}$ T. Their ages ranged from 5-77 years, with a mean age of 44. The male-to-female ratio was 7:6. The majority of wounds were located on the upper limb (46\%). Unfortunately, the site of the wound was not indicated in $31 \%$ of cases.

\section{Microbiological findings}

Single organisms were isolated in the majority of cases $(62 \%, \mathrm{n}=8)$. Six of the cases were proved to have $S$. aureus infections, while the remaining three were infected with $S$. pyogenes. Isolates did not show any bacterial growth in two cases, which may have been because of initiation of antibiotic therapy prior to microbiological sampling, or may have represented true spider bite cases. Mixed bacterial cultures were obtained, showing Klebsiella pneumoniae, Eschericiae coli, Proteus mirabilis and coagulase-negative Staphylococcus spp. in the remaining three cases (Table I).

In general, all isolates were highly sensitive and no cases of methicillin-resistant $S$. aureus were isolated. S. pyogenes

Table I. Demographic and microbiological information of patients presenting with an alleged spider bite

\begin{tabular}{|c|c|c|c|c|c|c|c|c|c|c|c|c|}
\hline Case & Age & Sex & Site & Sampling & Isolate & $\begin{array}{l}\text { Positive } \\
\text { isolates }\end{array}$ & $P$ and $A$ & E & Clin & Clox & Gent & Co-amox \\
\hline 1 & 77 & M & Sacrum & $T \times 1$ & S. aureus & 1 & $\mathrm{R}$ & S & $S$ & S & ND & ND \\
\hline 2 & 42 & M & Hand & PS $\times 1$ & S. aureus & 1 & $\mathrm{R}$ & S & $S$ & $S$ & ND & ND \\
\hline 3 & 32 & $M$ & Unknown & PS $\times 1$ & S. aureus & 1 & $\mathrm{R}$ & S & S & $S$ & ND & ND \\
\hline 4 & 44 & $\mathrm{~F}$ & Unknown & PS $\times 1$ & S. aureus & 1 & R & S & $S$ & $S$ & ND & ND \\
\hline \multirow{2}{*}{5} & \multirow{2}{*}{48} & \multirow{2}{*}{ M } & \multirow{2}{*}{ Leg } & PS $\times 3$ & S. aureus & 3 & $\mathrm{R}$ & S & $S$ & $S$ & ND & ND \\
\hline & & & & $T \times 3$ & S. aureus & 3 & $\mathrm{R}$ & S & $S$ & $S$ & ND & ND \\
\hline 6 & 70 & M & Knee & PS $\times 3$ & S. aureus & 3 & R & S & $S$ & $S$ & ND & ND \\
\hline 7 & 48 & $\mathrm{~F}$ & Hand & PS $\times 3$ & AHS group $A$ & 2 & $S$ & $S$ & ND & ND & ND & ND \\
\hline 8 & 30 & $\mathrm{~F}$ & Unknown & PS $x 1$ & AHS group A & 1 & S & ND & ND & ND & ND & ND \\
\hline \multirow{3}{*}{9} & \multirow{3}{*}{29} & \multirow{3}{*}{$\mathrm{F}$} & \multirow{3}{*}{ Axilla } & \multirow{3}{*}{$T \times 2$} & S. aureus & 2 & $\mathrm{R}$ & S & $S$ & ND & ND & ND \\
\hline & & & & & K. pneumoniae & 2 & $\mathrm{R}$ & ND & ND & $S$ & S & $S$ \\
\hline & & & & & E. coli & 2 & $S$ & ND & ND & S & S & S \\
\hline 10 & 49 & M & Hand & $T \times 1$ & P. mirabilis & 1 & $S$ & ND & ND & ND & S & S \\
\hline \multirow{2}{*}{11} & \multirow{2}{*}{36} & \multirow{2}{*}{ M } & \multirow{2}{*}{ Arm } & \multirow{2}{*}{$T \times 1$} & CNS & 1 & $\mathrm{R}$ & $\mathrm{R}$ & $\mathrm{R}$ & $\mathrm{R}$ & ND & ND \\
\hline & & & & & E. coli & 1 & $\mathrm{R}$ & ND & ND & ND & $\mathrm{R}$ & $\mathrm{S}$ \\
\hline 12 & 5 & $\mathrm{~F}$ & Unknown & $T \times 2$ & $N G$ & 0 & $\mathrm{~N} / \mathrm{A}$ & $\mathrm{N} / \mathrm{A}$ & $\mathrm{N} / \mathrm{A}$ & $\mathrm{N} / \mathrm{A}$ & N/A & $\mathrm{N} / \mathrm{A}$ \\
\hline 13 & 49 & $\mathrm{~F}$ & Arm & $T \times 1$ & $N G$ & 0 & NA & $N / A$ & $\mathrm{~N} / \mathrm{A}$ & $\mathrm{N} / \mathrm{A}$ & $N / A$ & $\mathrm{~N} / \mathrm{A}$ \\
\hline
\end{tabular}

AHS group A: Streptococcus pyogenes or group A beta-haemolytic Streptococcus, Clin: clindamycin, Clox: cloxacillin, Co-amox: co-amoxyclav, CNS: coagulase-negative Staphylococcus, E: erythromycin, E. coli: Eschericiae coli, F: female, Gent: gentamicin, K. pneumoniae: Klebsiella pneumoniae, M: male, N/A; not applicable, ND: sensitivity not determined, NG: no bacterial growth, P and A: penicillin and ampicillin, P. mirabilis: Proteus mirabilis, PS: pus swab, R: resistant, S: sensitive, S. aureus: Staphylococcus aureus, T: tissue

Cases 1 to 8 are likely to represent primary soft tissue infection

Cases 9 to 11 represent more complicated cases, with mixed infection

Cases 12 and 13 may have yielded no bacterial growth because of initiation of antibiotic therapy prior to microbiological sampling, or they may represent true spider bite cases 
isolates are not routinely subject to sensitivity testing, as no resistant isolates have ever been described. Therefore, penicillin therapy is considered to be adequate. ${ }^{5}$ However, as the testing was performed in an academic laboratory, surveillance sensitivities were performed.

\section{Discussion}

Only four medically important spiders have been described in South Africa. These include the neurotoxic Lactrodectus spp. (black widow or button spider), and three cytotoxic spiders, Cheiracanthium spp. (sac spider), Loxosceles spp. (violin spider, brown spider or brown recluse spider) and Sicarius spp. (sixeyed crab spider). ${ }^{6}$ In a case series performed by Newlands et al in which 40 cases of spider bites were examined, these genera were found in the Gauteng area, with the exception of the Sicarius spp. ${ }^{6}$ Although cytotoxic spider bites lead to local tissue destruction, the violin spider has been the most commonly implicated, particularly in studies performed in the USA. ${ }^{7,8}$ when comparing American and South African studies, a striking difference in the history of true spider bites is the distinct lack of systemic symptoms in local cases. ${ }^{9}$ This has not been attributed to reduced toxicity of the venom, but rather to a reduction in toxin volume, owing to the smaller size of the spider. ${ }^{6}$ In the case series by Newlands et al, more than $20 \%$ of reported spider bite cases were bacterial infections. ${ }^{6}$

Typically, alleged spider bites have occurred in settings of community-acquired, methicillin-resistant $S$. aureus outbreaks. ${ }^{1,3,7,8,10}$ It is likely that attributing the wound to a spider bite occurs because the majority of these infections occur spontaneously ${ }^{10}$ in younger, healthier patients..$^{11-13}$ In addition, these infections are also typically found on the extremities, ${ }^{14}$ suggesting exposure to an insect. As the bites of Loxosceles and Cheiracanthium spp. are completely painless and superficial, ${ }^{6}$ the clinical course of a spider bite would be very similar to that of soft tissue infection with either $S$. aureus or $S$. pyogenes. In the current study, $S$. aureus cases of infection were attributed to methicillin-sensitive organisms, and not methicillin-resistant $S$. aureus, as clearly described in the international literature. ${ }^{7,8,10}$ Spontaneous skin and soft tissue infection frequently occurs due to infection with $S$. aureus and Streptococcus spp., and in particular S. pyogenes. ${ }^{5}$

Some authors have suggested the possibility that spiders inoculate microbes during their bite, resulting in subsequent positive cultures. ${ }^{1}$ However, an investigation of 100 common house spider specimens yielded very few positive cultures, and no cases cultured $S$. aureus. ${ }^{15}$ The general milieu on a spider is not likely to provide a suitable environment in which the presence of human pathogens is sustained. Therefore, it seems that the association of spontaneous skin and soft tissue infection with spider bites remains based on reputation, rather than evidence. An alternative theory would be that following a spider bite, secondary infection with skin flora occurs. Although $S$. aureus and $S$. pyogenes are not considered to be normal skin flora, these organisms may be present on the skin, leading to secondary infection.
The diagnosis of a spider bite is often made incorrectly. Cases of lymphoma, basal-cell carcinoma and pyoderma gangrenosum have also been misdiagnosed as such initially. ${ }^{3}$ An array of infectious diseases can be included in the differential diagnosis, including herpes infection, sporotrichosis, syphilis and Chagas disease ${ }^{16}$ as a bite wound in the lesion ${ }^{6}$ is infrequently established, and visualisation of the spider prior to symptoms ${ }^{17,18}$ seldom confirmed. Therefore, a diagnosis of a spider bite should be one of exclusion, that follows a thorough history and clinical examination. In general, clinical complaints of spider bites by patients should be regarded as a nondescript spontaneous skin and soft tissue infection with dermonecrosis. ${ }^{1,19}$

\section{Declaration}

No benefits of any form have been received, or will be received, from a commercial party, that either directly or indirectly relate to the subject of this article.

The work of Dr Hilgaard Visser and Dr Adele Visser is financially supported by the Discovery Foundation.

\section{References}

Suchard J. "Spider bite" lesions are usually diagnosed as skin and soft-tissue infections. J Emerg Med. 2011;41(5):473-481.

2. Russell F, Gertsch W. For those who treat spider or suspected spider bites. Toxicon 1983;21(3):337-339.

3. Vetter R, Pagac B, Reiland R, et al. Skin lesions in barracks: consider communityacquired methicillin-resistant Staphylococcus aureus infection instead of spider bites. Mil Med. 2006;171(9):830-832.

4. Clinical and Laboratory Standards Institute. Performance standards for antimicrobial susceptibility testing. Wayne: CLSI; 2006.

5. Jones M, Karlowsky J, Draghi D, et al. Epidemiology and antibiotic susceptibility of bacteria causing skin and soft tissue infections in the USA and Europe: a guide to appropriate antimicrobial therapy. Int J Antimicrob Agents. 2003;22(4):406-419.

6. Newlands G, Atkinson P. Review of southern African spiders of medical importance, with notes on signs and symptoms of envenomation. S Afr J Med. 1988;73(4):235-239.

7. Dominguez T. It's not a spider bite, it's community-acquired methicillin-resistant Staphylococcus aureus. J Am Board Fam Pract. 2004;17(3):220-225

8. Rogers K, Klotz C, Jack M, Seger D. Systemic loxoscelism in the age of communityacquired methicillin-resistant Staphylococcus aureus. Ann Emerg Med. 2011;57(2):138-140.

9. Wasserman G, Anderson P. Loxoscelism and necrotic arachnidism. J Toxicol Clin Toxicol 1984;21-(24-25):451-472.

10. Vozdecky C. Community-acquired methicillin-resistant Staphylococcus aureus: not just a spider bite. Fam Comm Health. 2009;32(1):76-84

11. Hota B, Ellenbogen C, Hayden M, et al. Community-associated methicillin-resistant Staphylococcus aureus skin and soft tissue infections at a public hospital: do public housing and incarceration amplify transmission? Arch Int Med. 2007:167(10):1026-1033.

12. Jacobus C, Lindsel C, Leach S, et al. Prevalence and demographics of methicillin resistant Staphylococcus aureus in culturable skin and soft tissue infections in an urban emergency department. BMC Emerg Med. 2007;7:19.

13. Moran G, Krishnadasan A, Gorwitz R. Methicillin-resistant $\mathrm{S}$ aureus infections among patients in the emergency department. N Engl J Med. 2006;355(7):666-674

14. Frazee $B$, Lynn J, Charlebois $\mathrm{E}$, et al. High prevalence of methicillin-resistant Staphylococcus aureus in emergency department skin and soft tissue infections. Ann Emerg Med. 2005;45(3):311-320.

15. Baxtrom C, Mongkolpradit T, Kasimos J, et al. Common house spiders are not likely vectors of community-acquired methicillin-resistant Staphylococcus aureus infections. J Med Entomol. 2006;43:962-965.

16. Diaz J, Leblanc K. Common spider bites. Am Fam Physician. 2007;75(6):869-873.

17. Isbister G. Necrotic arachnidism: the mythology of a modern plague. Lancet. 2004:364(9433):484-453.

18. Vetter R. Myths about spider envenomation and necrotic skin lesions. Lancet. 2004;364(9433):484-485.

19. Vetter R, Bush S. The diagnosis of brown recluse spider bite is overused for dermonecrotic wounds of uncertain etiology. Ann Emerg Med. 2002;39(5):544-546. 